\title{
Applicability of Dickson Charge Pump in Energy Harvesting Systems: Experimental Validation of Energy Harvesting Charge Pump Model
}

\author{
Davor VINKO \\ Dept. of Communications, Faculty of Electrical Engineering, Computer Science and Information Technology Osijek, \\ J. J. Strossmayer University of Osijek, Kneza Trpimira 2b, 31000 Osijek, Croatia \\ davor.vinko@etfos.hr
}

Submitted January 16, 2017 / Accepted December 12, 2017

\begin{abstract}
Energy harvesting methods provide very low instantaneous power. Accordingly, available voltage levels are low and must be increased so that an energy harvesting method can be used as a power supply. One approach uses charge pumps to boost low AC voltage from energy harvester to a higher DC voltage. Characterized by very low output current and a wide span of operating frequencies, energy harvesting methods introduce a number of limitations to charge pump operation. This paper describes and models behavior of Dickson charge pump in energy harvesting applications. Proposed Energy Harvesting model is evaluated and compared with Standard and Tanzawa charge pump models and with measurement results. Based on the proposed model, the conditions that need to be satisfied so that a charge pump can reach maximum power point of energy harvesting system are defined. Parameter selection method optimized for maximum power point is presented and is experimentally validated.
\end{abstract}

\section{Keywords}

Charge pumps, energy harvesting, circuit analysis, mathematical model, maximum power point

\section{Introduction}

To use energy harvesting methods as a power supply, a mediator circuit is needed between the energy harvester and the load circuit. Energy harvester can provide a very low instantaneous power from various harvesting methods: RF, solar, wind, vibration, thermoelectric, etc. Power generally ranges from $1 \mu \mathrm{W}$ to $5 \mathrm{~mW}$ [1-3]. The generated voltage values are low and need to be boosted to a higher level. Energy harvesting methods can be divided into two groups: those that generate DC voltage and those that generate $\mathrm{AC}$ voltage. For energy harvesting methods that generate $\mathrm{AC}$ voltage (e.g. RF, vibration, wind, etc.), charge pumps can be directly used to boost low AC voltage from energy harvester to a higher DC voltage [4-6]. On the other hand, energy harvesting methods that generate DC voltage (e.g. solar, thermoelectric, etc.) need an additional low-power oscillator circuit to drive the charge pump circuit [7-10]. Therefore, in this paper, the generalized representation of an energy harvester will be an AC voltage source (as in Fig. 1), which also implies DC energy harvesters and associated oscillator circuits. There are numerous paper modeling and optimizing charge pump performance [11-14], but very few that analyze charge pump performance in energy harvesting applications. Designing and optimizing a charge pump for an energy harvesting application is challenging due to limitations caused by low voltage and limited current drivability of the energy harvester [15-17]. The charge pump design which is most often used in energy harvesting applications is the Dickson charge pump [18]. Some of the recently reported implementations of the Dickson charge pump in energy harvesting applications include photovoltaic [8], thermal [9], RF [19] and microbial fuel cell [10] energy harvesters. This paper proposes a generalized model of Dickson charge pump behavior in energy harvesting system.

Figure 1 shows Dickson charge pump used with energy harvester. Energy harvester is represented as a linear AC voltage source formed by ideal AC voltage source (with amplitude $U_{\mathrm{EH}}$ ) and series resistance $R_{\mathrm{EH}}$. Dickson charge pump is represented as a 4-stage charge pump with output capacitor $C_{\text {OUT }}$ equal to stage capacitors $C_{1}$ to $C_{4}$. Depending on the application, the diodes $D_{1}$ to $D_{5}$ can be discrete components (e.g. Schottky diodes) or diode-connected MOSFETs (in integrated circuits). Resistor $R_{\mathrm{LOAD}}$ represents a load circuit and $C_{\mathrm{PAR}}$ is a parasitic capacitance at charge pump input terminals. The main problem in charge pump design for an energy harvesting application is limited current drivability of the energy harvester. This limitation is not accurately modeled by standard charge pump models. There are three charge pump parameters (operating frequency $f$, number of charge pump stages $N$, and stage capacitor value $C$ ) that need to be chosen correctly to achieve a desired charge pump performance. In energy harvesting application, the operating frequency often depends on energy harvesting method used, and in 
most cases cannot be altered (without additional energy consumption), which leaves only two parameters to work with. There is a wide span of possible operating frequencies that energy harvesting methods provide, ranging from few $\mathrm{Hz}$ (kinetic energy harvesting), few $\mathrm{kHz}$ (vibration energy harvesting) up to $\mathrm{MHz}$ and $\mathrm{GHz}$ region (RF energy harvesting) [1], [6]. To successfully design a charge pump, a charge pump model must accurately describe charge pump behavior over wide span of operating frequencies and for very low input currents. This paper proposes an Energy Harvesting charge pump model which is compared to existing charge pump models and experimentally validated through measurements on laboratory prototype, Section 2. In Sec. 3 the maximum power point and efficiency are discussed and parameter selection method optimized for maximum power point is proposed. At the end of the paper, conclusion and references are given.

\section{Dickson Charge Pump Model}

Charge pump designing process starts by choosing initial parameter values: number of charge pump stages $N$, operating frequency $f$, capacitance of stage capacitors $C$. Combination of these three parameters $(N, f, C)$ must provide desired charge pump output voltage $U_{\text {OUT }}$ for a given energy harvester $\left(U_{\mathrm{EH}}, R_{\mathrm{EH}}\right)$, switch (diodes $D_{1}$ to $D_{5}$ in Fig. 1) threshold voltage $U_{\text {th }}$ and load resistance $R_{\text {LOAD }}$ (or output current $\left.I_{\text {OUT }}\right)$.

After the initial parameter $(N, f, C)$ values are chosen, they are fine-tuned through SPICE simulation. SPICE simulation is time consuming and is therefore used only for fine-tuning, whereas the initial parameters are chosen by a simpler (and less accurate) model. Standard Dickson charge pump model [20] is modeled as a linear DC voltage source with voltage $U_{0}$ and series resistance $R_{\mathrm{S}}$ (Fig. 1), which are given by (1) and (2), respectively.

$$
\begin{gathered}
U_{0 \_ \text {STANDARD_MODEL }}=(N+1) \cdot\left(U_{\mathrm{EH}}-U_{\mathrm{th}}\right)[\mathrm{V}], \\
R_{\text {S_STANDARD_MODEL }}=\frac{N}{f \cdot C}[\Omega] .
\end{gathered}
$$

Standard model does not include the current limitation of energy harvester and cannot accurately model the impact of limited input current. Energy harvester is modeled as a linear AC voltage source, $U_{\mathrm{EH}}$ and $R_{\mathrm{EH}}$ in Fig. 1. Series resistance $R_{\mathrm{EH}}$ corresponds to a current drivability of energy harvester, i. e. the lower current corresponds to a higher resistance $R_{\mathrm{EH}}$. In general, $R_{\mathrm{EH}}$ value ranges from few $\mathrm{k} \Omega$ up to several tens of $\mathrm{M} \Omega$ what corresponds to a current drivability of energy harvester of a few $\mathrm{mA}$ down to a hundred nA. Dickson charge pump model which deals with a limited input current due to a high output impedance of a voltage source is presented by Toru Tanzawa [21]. Tanzawa model is designed to model the behavior of a DC-DC voltage multiplier driven by a DC energy transducer such as photovoltaic and thermoelectric generators.

$$
\begin{aligned}
& U_{0_{-} \text {TANZAWA_MODEL }}=(N+1) \cdot\left(U_{\mathrm{EH}}-U_{\mathrm{th}}\right)[\mathrm{V}], \\
& R_{\mathrm{S}_{-} \text {TANZAWA_MODEL }}=(N+1)^{2} R_{\mathrm{EH}}+\frac{N}{f \cdot C}[\Omega] .
\end{aligned}
$$

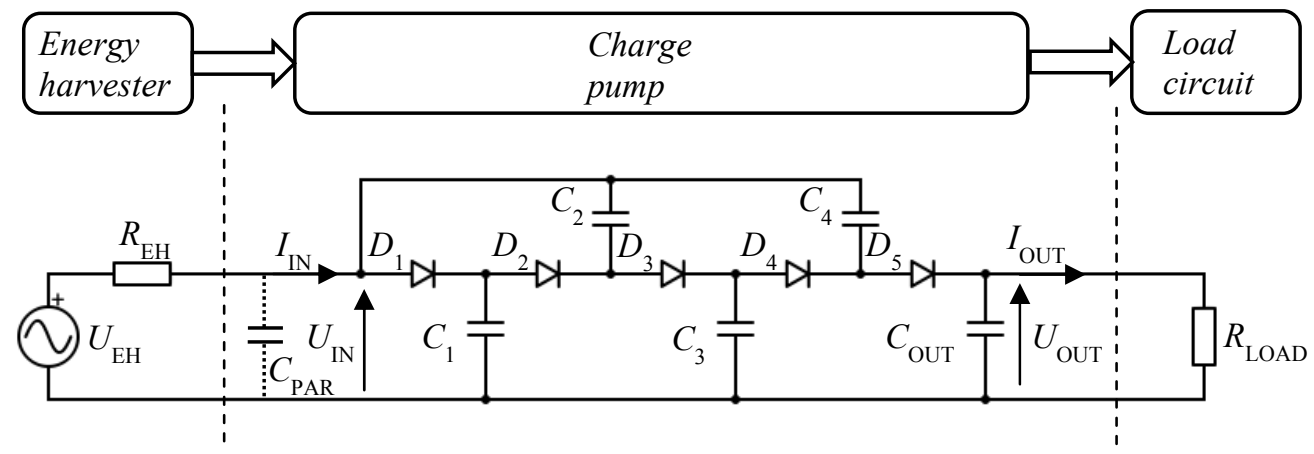

a) Dickson charge pump in energy harvesting system.

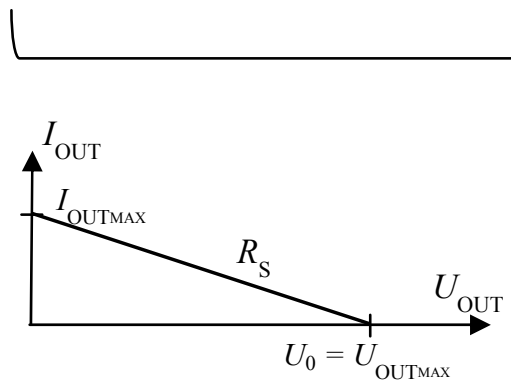

b) Output current-voltage characteristics.

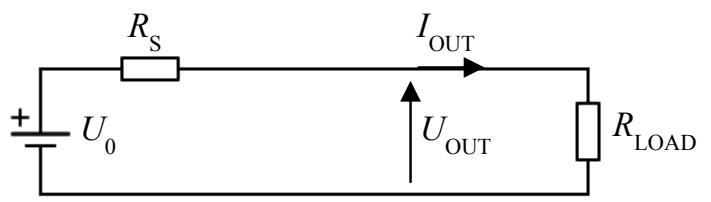

c) Energy harvester and charge pump equivalent model.

Fig. 1. Dickson charge pump powered from energy harvester. 


\subsection{Energy Harvesting Model}

Using the same methodology as presented in [21], charge pump powered from energy harvester can be modeled. The equivalent series resistance of the charge pump is affected by the number of charge pump stages $N$, stage capacitor value $C$, operating frequency $f$ and limitations caused by energy harvester. The impact of the first three parameters is modeled by standard model (2) and the impact of energy harvester $\left(U_{\mathrm{EH}}, R_{\mathrm{EH}}\right)$ can be evaluated separately and then added to the standard model.

There is a significant difference when a charge pump is driven by a source with square voltage waveform, as in [21], and when the charge pump is driven by an energy harvester with sine voltage waveform. The switch threshold voltage $U_{\text {th }}$ affects the RMS value of charge pump input current. With short-circuited charge pump output, the charge pump input draws maximal current from energy harvester. For a source with square voltage waveform, the switch threshold voltage $U_{\text {th }}$ decreases peak voltage value, (Fig. 2), and the maximal current from energy harvester equals:

$$
I_{\mathrm{EH}_{\mathrm{MAX}}}(\text { square })=\frac{U_{\mathrm{EH}}-U_{\text {th }}}{R_{\mathrm{EH}}}[\mathrm{A}] .
$$

With sine voltage waveform, the decrease in peak voltage value also causes the "dead" time $T_{\mathrm{OFF}}$, Fig. $2 \mathrm{~b}$. $T_{\mathrm{OFF}}$ is given by (6), where $T$ is a period of the sine wave. There are four $T_{\mathrm{OFF}}$ occurrences during one period which decreases the duty cycle $D$ of the signal (7). This decreases RMS value of the waveform, resulting in larger crest factor value $F$ compared to crest factor of sine waveform (8) (detailed derivation is given in Appendix).

$$
\begin{gathered}
T_{\mathrm{OFF}}=\frac{\arcsin \left(\frac{U_{\mathrm{th}}}{U_{E H}}\right)}{2 \pi} T[\mathrm{~s}], \\
D=1-4 \cdot \frac{T_{\mathrm{OFF}}}{T}[-], \\
F \approx \frac{\sqrt{2}}{D}[-] .
\end{gathered}
$$

For sine voltage waveform, the maximal RMS current then equals:

$$
I_{\mathrm{EH}_{\mathrm{MAX}}}=\frac{1}{F} \frac{U_{\mathrm{EH}}-U_{\text {th }}}{R_{\mathrm{EH}}}[\mathrm{A}] .
$$

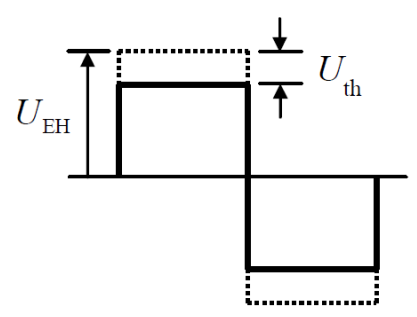

(a)

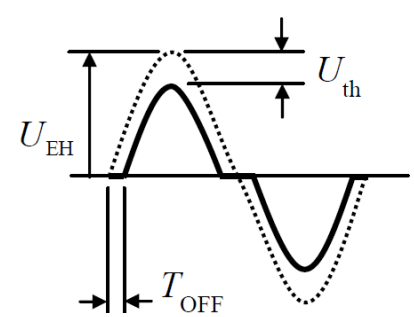

(b)
Fig. 2. Impact of switch threshold voltage $U_{\text {th }}$ on energy harvester voltage waveform.
Equation (9) represents the generalized expression for the maximal current from energy harvester. In case of square voltage waveform, the crest factor $F$ equals 1, which corresponds to (5). Relation between charge pump input current $I_{\mathrm{IN}}$ and output current $I_{\mathrm{OUT}}$ is defined by the number of charge pump stages:

$$
I_{\mathrm{OUT}}=\frac{I_{\mathrm{IN}}}{N+1}[\mathrm{~A}] \text {. }
$$

Therefore, by inserting (9) in (10) as $I_{\mathrm{IN}}$, the maximal charge pump output current equals [21]:

$$
I_{\mathrm{OUT}_{\mathrm{MAX}}}=\frac{1}{F} \frac{U_{\mathrm{EH}}-U_{\mathrm{th}}}{(N+1) \cdot R_{\mathrm{EH}}}[\mathrm{A}] .
$$

Maximal charge pump output voltage equals $U_{0}$ in both Standard (1) and Tanzawa (3) model:

$$
U_{\mathrm{OUT}_{\mathrm{MAX}}}=U_{0}=(N+1) \cdot\left(U_{\mathrm{EH}}-U_{\mathrm{th}}\right)[\mathrm{V}] .
$$

The equivalent series resistance $R_{\mathrm{S}}$, due to limitation caused by energy harvester, can be expressed by dividing (12) by (11), as follows:

$$
R_{\mathrm{S}}=F \cdot(N+1)^{2} \cdot R_{\mathrm{EH}}[\Omega] .
$$

By expanding the Standard model (2) with (13), the equivalent series resistance $R_{\mathrm{S}}$ of a charge pump, powered by an energy harvester, can be described as:

$$
R_{\mathrm{S}_{-} \mathrm{EH}}=F \cdot(N+1)^{2} \cdot R_{\mathrm{EH}}+\frac{N}{f \cdot C}[\Omega] .
$$

With very low input current and wide span of operating frequencies, parasitic capacitance at charge pump input terminals $\left(C_{\mathrm{PAR}}\right.$ in Fig. 1$)$ plays a significant role in charge pump performance. This parasitic capacitance at device input terminals must be charged and discharged in each operating cycle, and its value depends on design and process parameters. Together with resistance $R_{\mathrm{EH}}$ it forms a low-pass filter at charge pump input which decreases voltage amplitude at charge pump input terminals $\left(U_{\mathrm{IN}}\right)$. Therefore, maximal charge pump output voltage $U_{0}$ in Energy Harvesting model is expressed by (15), whereas the series resistance $R_{\mathrm{S}}$ equals (14).

$$
U_{0_{-} \mathrm{EH}}=(N+1)\left(\frac{U_{\mathrm{EH}}}{\sqrt{R_{\mathrm{EH}}^{2} \omega^{2} C_{\mathrm{PAR}}^{2}+1}}-U_{\mathrm{th}}\right)[\mathrm{V}] .
$$

\subsection{Model Validation}

Energy harvesting methods are characterized by very low output currents and wide span of possible operating frequencies. Therefore, the charge pump performance is evaluated in $f-R$ plane. $f-R$ plane shows the charge pump output value (e.g. output voltage, output power) for different values of operating frequency $f$ and load resistance $R_{\mathrm{LOAD}}$.

Experimental setup (Fig. 1 with parameter values given in Tab. 1) is realized with discrete components. 
Switches in the charge pump are implemented by Schottky diodes with forward voltage of $0.25 \mathrm{~V}\left(U_{\mathrm{th}}\right)$. Experimental setup has a parasitic input capacitance $C_{\mathrm{PAR}}$ of $30 \mathrm{pF}$. Energy harvester is implemented by a linear AC voltage source $\left(U_{\mathrm{EH}}\right)$ with series resistor $\left(R_{\mathrm{EH}}\right)$. Experimental setup is used to compare Standard model (1), (2), Tanzawa model (3), (4), and Energy Harvesting model (14), (15), with measurement results for charge pump output voltage. Due to the structure of mathematical expressions for $U_{0}$ voltage in all three models, the sensitivity of all there models to different input voltage is the same. Energy Harvesting model adds a low-pass filter to the expression but it affects only a frequency response of the model. Therefore, the input voltage is kept constant, as given in Tab. I. In each measurement of the $f-R$ plane the load resistance is varied from $10 \mathrm{k} \Omega$ to $10 \mathrm{M} \Omega$ (in 10 discrete steps) and the operating frequency is varied from $100 \mathrm{~Hz}$ to $10 \mathrm{MHz}$ (in 16 discrete steps) which gives the total of 160 test points for each $f-R$ plane. Results are given in Fig. 3 .

The Standard model (Fig. 3a) shows the greatest deviation with respect to measured results (Fig. 3d). Such behavior is expected since the Standard model does not take into account the impact of limited input current (due to $R_{\mathrm{EH}}$ ). Tanzawa model (Fig. 3b) models the impact of limited input current, but with significant error on higher frequencies, compared to measurement. Energy Harvesting model (Fig. 3c) gives the best fit to the measured results.

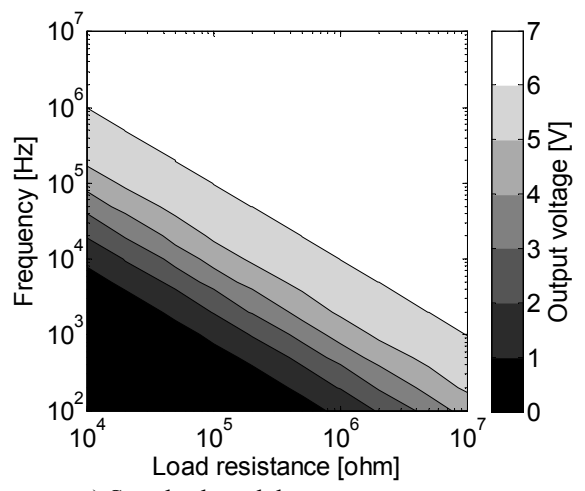

a) Standard model $R^{2}=-2.2143$

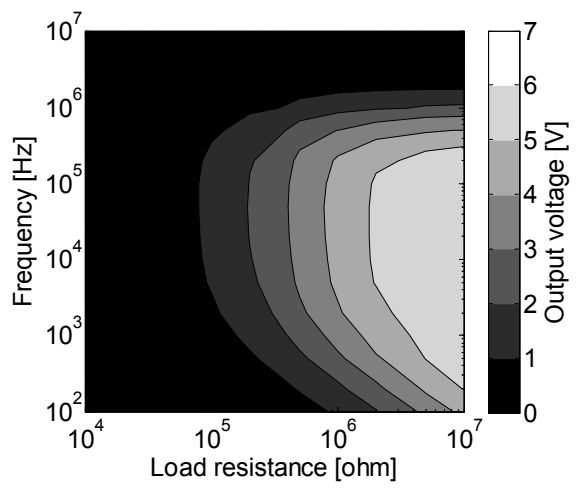

c) Energy Harvesting model $R^{2}=0.9904$

\begin{tabular}{|l|l|}
\hline Parameter & Value \\
\hline$f$ & $100 \mathrm{~Hz}-10 \mathrm{MHz}$ \\
\hline$R_{\mathrm{LOAD}}$ & $10 \mathrm{k} \Omega-10 \mathrm{M} \Omega$ \\
\hline$N$ & 4 \\
\hline$C$ & $10 \mathrm{nF}$ \\
\hline$U_{\mathrm{th}}$ & $0.25 \mathrm{~V}$ \\
\hline$C_{\mathrm{PAR}}$ & $30 \mathrm{pF}$ \\
\hline$U_{\mathrm{EH}}$ & $1.5 \mathrm{~V}$ \\
\hline$R_{\mathrm{EH}}$ & $10 \mathrm{k} \Omega$ \\
\hline
\end{tabular}

Tab. 1. Experimental setup: Parameter values for $f-R$ measurement.

The models are evaluated based on how accurately they model charge pump behavior in $f-R$ plane. To evaluate the goodness of fit of charge pump models with respect to measured results, a coefficient of determination $R^{2}(\mathrm{R}$ squared measure) is used (16):

$$
R^{2}=1-\frac{\sum_{i=1}^{n}\left(E_{i}-M_{i}\right)^{2}}{\sum_{i=1}^{n}\left(E_{i}-\frac{1}{n} \sum_{i=1}^{n} E_{i}\right)}[-]
$$

where $n$ is the number of test points per measurement, $E_{i}$ represents experimental values $\left(E_{1}\right.$ to $\left.E_{n}\right)$ and $M_{i}$ represents modeled values $\left(M_{1}\right.$ to $\left.M_{n}\right)$ for a given test point $(i=1$ to $n)$. The results are given in Fig. 3 under each model. $R^{2}$ confirms that the Energy Harvesting model gives the best fit to the measured results.
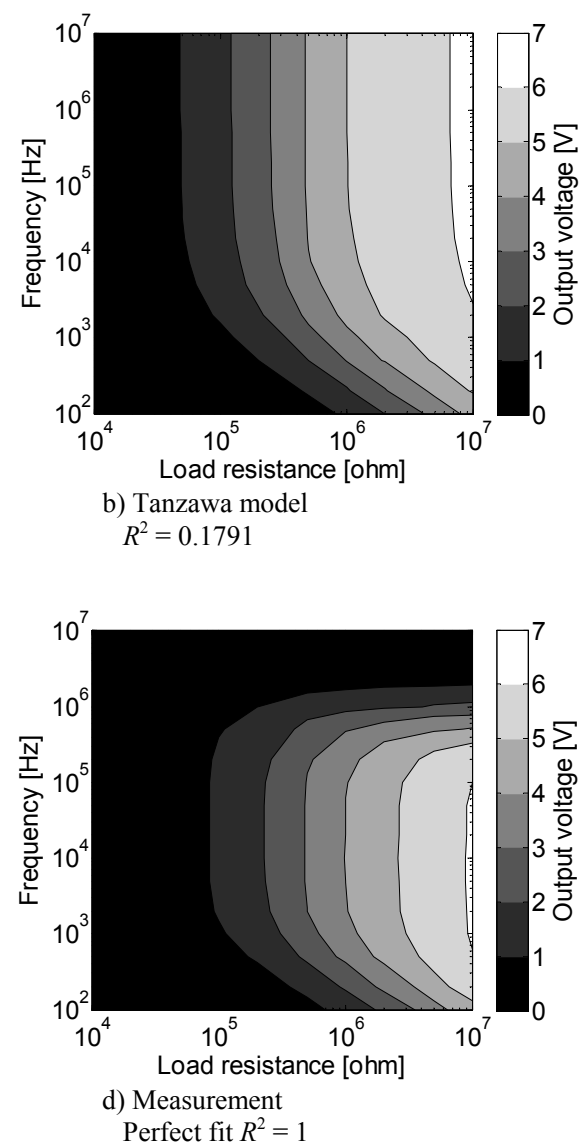

Fig. 3. Comparison of charge pump models and measurement results for charge pump output voltage in $f-R$ plane for parameter values given in Tab. 1. 


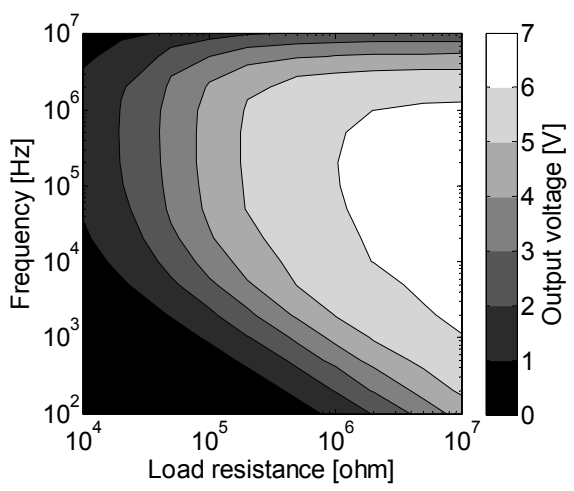

a) Energy Harvesting model $R^{2}=0.9782$

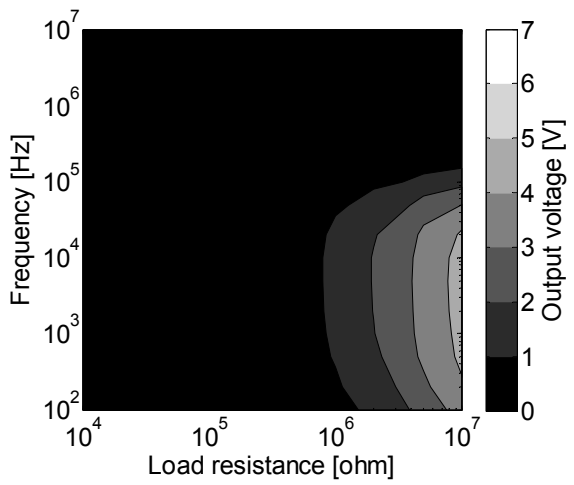

c) Energy Harvesting model $R^{2}=0.9808$

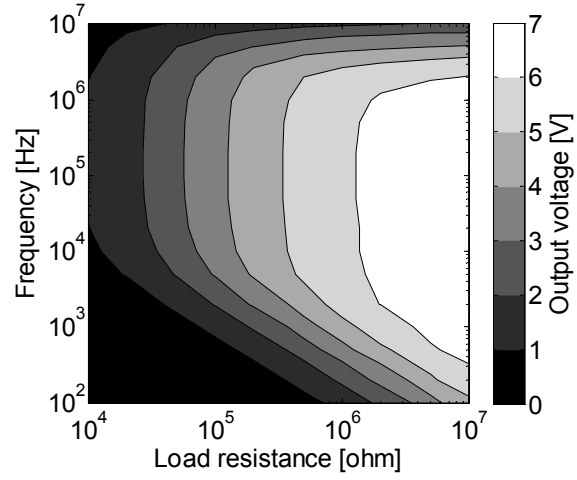

b) Measurement $R_{\mathrm{EH}}=1 \mathrm{k} \Omega$

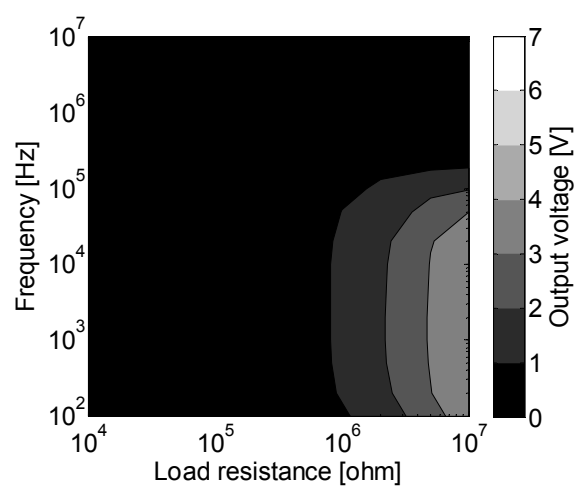

d) Measurement

Fig. 4. Comparison of Energy Harvesting model and measurement results for charge pump output voltage in $f-R$ plane for different values of $R_{\mathrm{EH}}$, with respect to Tab. 1 .

For further validation, Energy Harvesting model is compared to experimental results for different values of $R_{\mathrm{EH}}$, Fig. 4. All parameters are as in Tab. 1, except $R_{\mathrm{EH}}$ values which are given in Fig. 4. Results show that with higher $R_{\mathrm{EH}}$ resistance (lower instantaneous current available from energy harvester) the output voltage decreases significantly.

\section{Maximum Power Point}

In energy harvesting systems the goal is to extract as much power from energy harvester as possible. Charge pump parameters should be optimized for maximum power point (MPP). Figure 5 shows measurement results for charge pump output power in $f-R$ plane. The experimental setup is as given in Tab. 1, with changed parameters marked in Fig. 5. Output power measurements are done for different values of $R_{\mathrm{EH}}$ (Fig. 5a, b, c) which correspond to output voltage measurements given by Fig. $4 \mathrm{~b}$, Fig. $3 \mathrm{~d}$ and Fig. 4d, respectively. In addition, the output power is also measured for lower stage capacitance $C$ value (Fig. 5d) and higher number of charge pump stages $N$ (Fig. 5e), with respect to Tab. 1 .

Maximal output power $P_{\text {MAX }}$ (values are given under each measurement in Fig. 5) is directly affected by $R_{\mathrm{EH}}$ value. With $R_{\mathrm{EH}}$ increase from $1 \mathrm{k} \Omega$ to $100 \mathrm{k} \Omega, P_{\mathrm{MAX}}$ decreases from $166 \mu \mathrm{W}$ to $1.925 \mu \mathrm{W}$. Changes in stage capacitor size $C$ and number of stages $N$, result with less than $10 \%$ change in $P_{\text {MAX }}$ value. With charge pump modeled as a linear DC voltage source, maximal power is delivered to the load when charge pump output voltage drops to $50 \%$ of maximal charge pump output voltage. Energy Harvesting model is used to calculate the dotted line for each measurement (Fig. 5.), which marks the line in $f-R$ plane where the charge pump output voltage equals $50 \%$ of maximal output voltage value. The modeled (dotted) line passes through area with maximal measured output power in $f-R$ plane. This line can be divided into three sections, Fig. $5 \mathrm{f}$. Section 1 is defined by the upper cut-off frequency:

$$
f=\frac{1}{2 \pi R_{\mathrm{EH}} C_{\mathrm{PAR}}}[\mathrm{Hz}] .
$$

For sections 2 and 3 output load $R_{\mathrm{LOAD}}$ equals charge pump series resistance $R_{\mathrm{S}}$ (14). In section 2 , the dominant part of $R_{\mathrm{S}}$ is $F(N+1)^{2} R_{\mathrm{EH}}$, whereas in section 3 the dominant part is $N / f C$. The maximal measured output power corresponds to section 2 , where $R_{\mathrm{S}}$ has its minimal value:

$$
R_{\mathrm{LOAD}}=R_{\mathrm{S}_{-} \min }=F \cdot(N+1)^{2} R_{\mathrm{EH}}[\Omega] .
$$




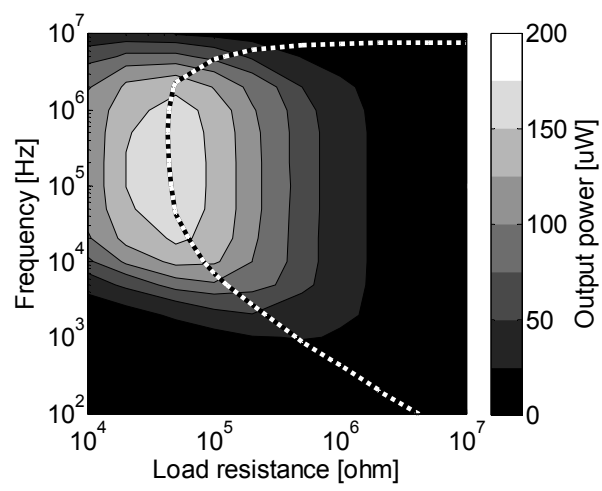

a) $R_{\mathrm{EH}}=1 \mathrm{k} \Omega \quad P_{\mathrm{MAX}}=166 \mu \mathrm{W}$

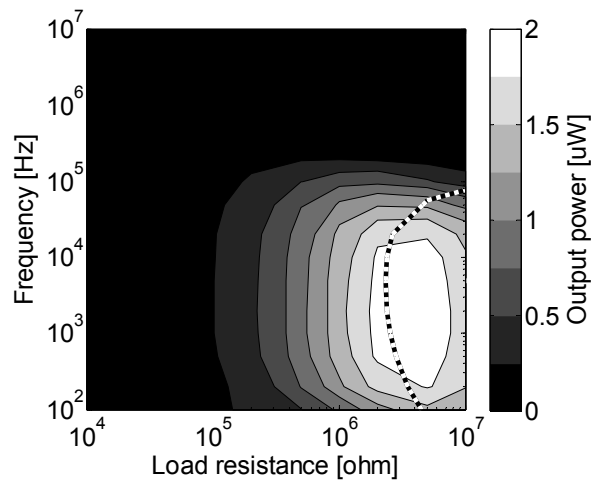

c) $R_{\mathrm{EH}}=100 \mathrm{k} \Omega \quad P_{\mathrm{MAX}}=1.925 \mu \mathrm{W}$

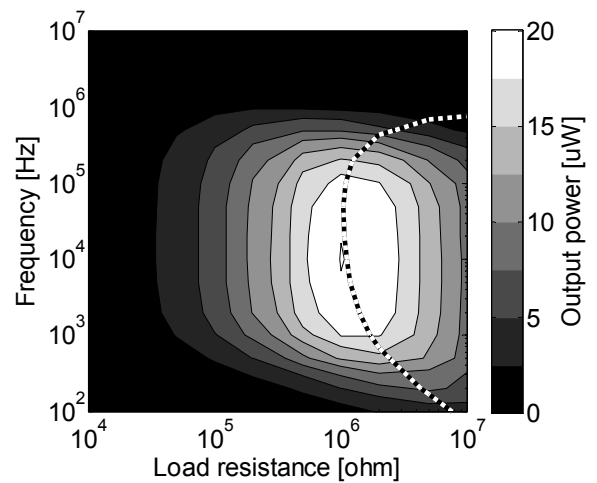

e) $N=8 \quad P_{\text {MAX }}=20.07 \mu \mathrm{W}$

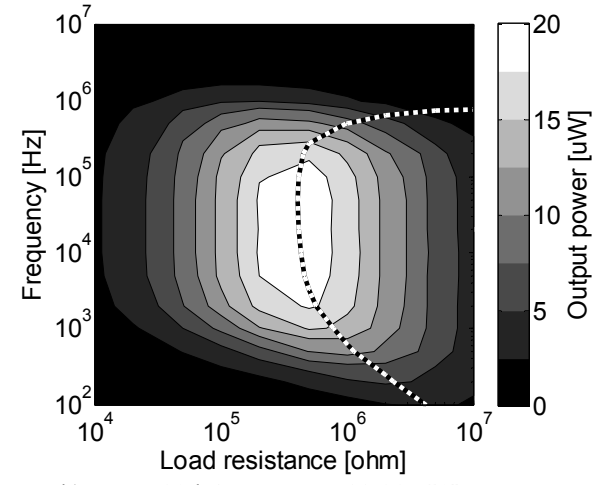

b) $R_{\mathrm{EH}}=10 \mathrm{k} \Omega \quad P_{\mathrm{MAX}}=18.99 \mu \mathrm{W}$
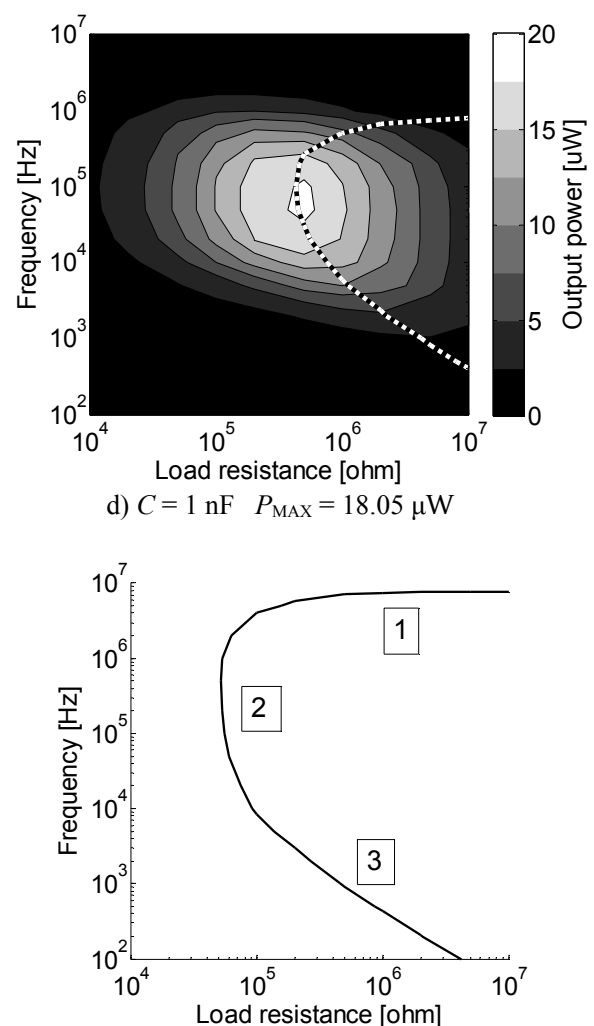

f) $P_{\text {MAX }}$ line

Fig. 5. Charge pump output power measurement for different values of $R_{\mathrm{EH}}, C$ and $N$, with respect to Tab. 1

\begin{tabular}{|c|c|c|c|c|c|c|c|c|c|c|}
\hline \multicolumn{7}{|c|}{ Test point parameters at measured MPP } & \multicolumn{4}{|c|}{$P_{\mathrm{MAX}}[\mu \mathrm{W}] ; \eta$} \\
\hline Fig. & $\begin{array}{c}R_{\mathrm{EH}} \\
{[\mathrm{k} \Omega]}\end{array}$ & $\begin{array}{c}N \\
{[-]}\end{array}$ & $\begin{array}{c}C \\
{[\mathrm{nF}]}\end{array}$ & $\begin{array}{c}f \\
{[\mathrm{kHz}]}\end{array}$ & $\begin{array}{c}R_{\mathrm{LOAD}} \\
{[\mathrm{k} \Omega]}\end{array}$ & $P_{\mathrm{EHmax}}$ & Measured & EH model & $\begin{array}{c}\text { Tanzawa } \\
\text { model }\end{array}$ & $\begin{array}{c}\text { Standard } \\
\text { model }\end{array}$ \\
\hline $5 \mathrm{a}$ & 1 & 4 & 10 & 200 & 47 & $281.25 ; 1$ & $166 ; 0.59$ & $232.4 ; 0.826$ & $329.4 ; 1.171$ & $722.3 ; 2.568$ \\
\hline $5 \mathrm{~b}$ & 10 & 4 & 10 & 20 & 470 & $28.125 ; 1$ & $18.99 ; 0.675$ & $22.29 ; 0.793$ & $31.29 ; 1.113$ & $67.00 ; 2.382$ \\
\hline $5 \mathrm{c}$ & 100 & 4 & 10 & 2 & 4700 & $2.8125 ; 1$ & $1.925 ; 0.684$ & $2.324 ; 0.826$ & $3.294 ; 1.171$ & $7.200 ; 2.560$ \\
\hline $5 \mathrm{~d}$ & 10 & 4 & 1 & 50 & 470 & $28.125 ; 1$ & $18.05 ; 0.642$ & $24.03 ; 0.854$ & $34.65 ; 1.232$ & $77.90 ; 2.770$ \\
\hline $5 \mathrm{e}$ & 10 & 8 & 10 & 10 & 1000 & $28.125 ; 1$ & $20.07 ; 0.714$ & $22.66 ; 0.806$ & $35.43 ; 1.260$ & $100.9 ; 3.588$ \\
\hline
\end{tabular}

Tab. 2. Charge pumps output power and efficiency at maximum power point.

\subsection{Parameter Selection Method}

The model should give a simple and fairly accurate method on how to choose initial charge pump parameters $(N, C)$ for a known energy harvester $\left(U_{\mathrm{EH}}, R_{\mathrm{EH}}, f\right)$ and load circuit ( $U_{\mathrm{OUT}}, R_{\mathrm{LOAD}}$ ). The required number of charge pump stages $N$ depends on the difference between energy harvester voltage amplitude $U_{\mathrm{EH}}$ and maximal charge pump output voltage $U_{0},(1)$. To achieve maximum power point, $U_{0}$ should be two times higher than the desired output voltage $U_{\text {OUT }}$, thus $N$ equals:

$$
N=2\left(\frac{U_{0}}{U_{\mathrm{EH}}-U_{\text {th }}}-1\right)[-] .
$$


When the number of stages $N$ is defined, two conditions must be satisfied to use Dickson charge pump in an energy harvesting system. First condition, the operating frequency of energy harvester must be lower than the upper cut-off frequency (17). Second condition, to place charge pump operating point in $f$ - $R$ plane at maximum power point (MPP), the load resistance $R_{\mathrm{LOAD}}$ should match the minimal value of series resistance $R_{\mathrm{S}}$, (18). Both conditions are directly affected by the $R_{\mathrm{EH}}$ value. With $R_{\mathrm{EH}}$ increase, the upper cut-off frequency lowers and the equivalent series resistance $R_{\mathrm{S}}$ increases. If conditions are not met, charge pump operating point in $f-R$ plane cannot be placed at the maximum power point. If both conditions are satisfied, the stage capacitor value $C$ can be selected, keeping in mind that $F(N+1)^{2} R_{\mathrm{EH}}$ must be significantly larger than $N / f C$.

$$
C>\frac{N}{f \cdot F \cdot(N+1)^{2} R_{\mathrm{EH}}}[\mathrm{F}] .
$$

\subsection{Efficiency}

Charge pump efficiency $\eta$ is affected by parasitic input capacitance $C_{\mathrm{PAR}}$, parasitic capacitance $\left(C_{\mathrm{S}}\right)$ at the bottom-plate of each stage capacitor, switch "on" resistance $R_{\mathrm{ON}}$ and switch threshold voltage $U_{\text {th }}$. Switch "on" resistance is several orders of magnitude lower than output resistance of energy harvester $R_{\mathrm{EH}}$ and it can be neglected for most energy harvesting applications. Bottom-plate parasitic capacitance $C_{\mathrm{S}}$ has significant impact on efficiency in integrated charge pump circuits [12], [22], but its impact is not prominent for a charge pump realized with discrete components. Parasitic input capacitance $C_{\mathrm{PAR}}$ affects the efficiency of power transfer from energy harvester to the charge pump, whereas the threshold voltage $U_{\text {th }}$ affects the efficiency of the charge pump. At maximum power point which corresponds to section 2 of the $P_{\text {MAX }}$ line (Fig. 5f), threshold voltage $U_{\text {th }}$ has the dominant effect on charge pump efficiency, whereas the effect input capacitance $C_{\text {PAR }}$ is less prominent.

Table 2 gives measured and modeled values for maximal output power $P_{\mathrm{MAX}}$ and for charge pump efficiency $\eta$, corresponding to measurements given in Fig. 5. For each measurement, Table 2 gives the test point parameters at measured MPP. Standard model, Tanzawa model and Energy Harvesting model are compared to measurement results. $P_{\mathrm{MAX}}$ value for all three charge pump models is calculated at measured maximum power point. All three charge pump models result with higher values of maximal output power at MPP, with proposed model being the most accurate. Charge pump efficiency is evaluated with respect to maximal achievable output power from an energy harvester. At MPP, output power of $100 \%$ efficient charge pump $(\eta=1)$ would equal to maximal output power of energy harvester $P_{\text {EHmax }}$ :

$$
P_{\mathrm{EH} \max }=\frac{1}{4} \cdot \frac{\left(\frac{U_{\mathrm{EH}}}{\sqrt{2}}\right)^{2}}{R_{\mathrm{EH}}}=\frac{U_{\mathrm{EH}}{ }^{2}}{8 R_{\mathrm{EH}}}[\mathrm{W}] .
$$

The efficiency values given in Tab. 2 show that all three models result with higher than measured efficiency. Standard and Tanzawa model result with efficiency that is even higher than 1 . This is due to insufficiently accurate modeling of limited input current. The discrepancies between measured and modelled values are also present with Energy Harvesting model. This is due to assumption on which all models are based on. Models presume constant threshold voltage value $U_{\text {th }}$. In reality, threshold voltage increases with increase in output current (i.e. higher output power) and decreases for lower output currents (i.e. lower output power).

\section{Conclusion}

The Energy Harvesting model of the Dickson charge pump is presented and experimentally validated. Results have shown that the Energy Harvesting model describes the behavior of the Dickson charge pump in energy harvesting applications with sufficient accuracy. For charge pump output voltage, the average error of the Energy Harvesting model is under $3 \%$, according to the $\mathrm{R}$ squared measure of goodness of fit. A maximum power point in $f-R$ plane is defined. The impact of charge pump parameters (operating frequency $f$, capacitor size $C$, number of charge pump stages $N$ ) on the location of the maximum power point is accurately modeled by the Energy Harvesting model. Based on the dynamics of the maximum power point and the limitations imposed by energy harvesting, the conditions that need to be satisfied so that a charge pump can be used in energy harvesting system are defined and a parameter selection method optimized for maximum power point is proposed. Charge pump efficiency is discussed and is experimentally investigated at maximum power point. The average error of the Energy Harvesting model is $26 \%$, while Tanzawa and Standard model have average error of $83 \%$ and $324 \%$, respectively. Modeled efficiency has a higher error compared to the error of modeled output voltage. This is due to the simplicity of the charge pump models as they are used to calculate initial charge pump parameters, which will be fine-tuned through simulations. For such application, the Energy Harvesting model has sufficient accuracy for a wide span of operating frequencies and load resistances, and its level-of-complexity is comparable to other evaluated charge pump models.

\section{References}

[1] ZAHID KAUSAR, A. S. M., REZA, A. W., SALEH, M. U., et al. Energizing wireless sensor networks by energy harvesting systems: scopes, challenges and approaches. Renewable and Sustainable Energy Reviews, 2014, vol. 38(C), p. 973-989. DOI: 10.1016/j.rser.2014.07.035

[2] BElleVille, M., FANET, H., FIORINI, P., et al. Energy autonomous sensor systems: state and perspectives of a ubiquitous sensor technology. In Proceedings of the 3rd International 
Workshop on Advances in Sensors and Interfaces IWASI. Trani (Italy), 2009, p. 134-138. DOI: 10.1109/IWASI.2009.5184783

[3] BELleVille, M., CANTATORE, E., FANET, H., et al. Energy autonomous systems: future trends in devices, technology, and systems. CATRENE Working Group on Energy Autonomous Systems 2008. Table 3, p. 20.

[4] TSAI, T. H., SHIU, B. Y., SONG, B. H. A self-sustaining integrated CMOS regulator for solar and HF RFID energy harvesting systems. IEEE Journal of Emerging and Selected Topics in Power Electronics, 2014, vol. 2, no. 3, p. 434-442. DOI: 10.1109/JESTPE.2014.2314479

[5] RABEN, H., BORG, J., JOHANSSON, J. Design of voltage multipliers for maximized DC generation in inductively coupled RFID tags. IEEE Transactions on Circuits and Systems I: Regular Papers, 2014, vol. 61, no. 11, p. 3309-3317. DOI: 10.1109/TCSI.2014.2327305

[6] HARB, A. Energy harvesting: state-of-the-art. Journal of Renewable Energy, 2011, vol. 36, p. 2641-2654. DOI: 10.1016/j.renene.2010.06.014

[7] LIU, X., SANCHEZ-SINENCIO, E. A highly efficient ultralow photovoltaic power harvesting system with MPPT for internet of things smart nodes. IEEE Transactions on Very Large Scale Integration (VLSI) Systems, 2015, vol. 23, no. 12, p. 3065-3075. DOI: $10.1109 /$ TVLSI.2014.2387167

[8] TAR, B., CILINGIROGLU, U. Nanowatt-scale power management for on-chip photovoltaic energy harvesting beacons. IEEE Journal on Emerging and Selected Topics in Circuits and Systems, 2014, vol. 4, no. 3, p. 284-291. DOI: 10.1109/JETCAS.2014.2337192

[9] ASHRAF, M., MASOUMI, N. A thermal energy harvesting power supply with an internal startup circuit for pacemakers. IEEE Transactions on Very Large Scale Integration (VLSI) Systems, 2016, vol. 24, no. 1, p. 26-37. DOI: 10.1109/TVLSI.2015.2391442

[10] CARREON-BAUTISTA, S., ERBAY, C., HAN, A., et al. An inductorless DC-DC converter for an energy aware power management unit aimed at microbial fuel cell arrays. IEEE Journal of Emerging and Selected Topics in Power Electronics, 2015, vol. 3, no. 4, p. 1109-1121. DOI: 10.1109/JESTPE.2015.2398851

[11] PAlUMBO, G., PAPPALARDO, D. Charge pump circuits: an overview on design strategies and topologies. IEEE Circuits and Systems Magazine, 2010, vol. 10, no. 1, p. 31-45. DOI: 10.1109/MCAS.2009.935695

[12] PAlUMBO, G., PAPPALARDO, D., GAIBOTTI, M. Charge pump circuits: power consumption optimization. IEEE Transactions on Circuits and Systems I: Fundamental Theory and Applications, 2002, vol. 49, no. 11, p. 1535-1542. DOI: 10.1109/TCSI.2002.804544

[13] PALUMBO, G., BARNIOL, N., BETHAOUI, M. Improved behavioural and design model of an $\mathrm{N}$-th order charge pump. IEEE Transaction on Circuits and Systems I: Fundamental Theory and Applications, 2000, vol. 47, no. 2, p. 264-268. DOI: $10.1109 / 81.828583$

[14] TANZAWA, T., TANAKA, T. A dynamic analysis of the Dickson charge pump circuit. IEEE Journal of Solid-State Circuits, 1997, vol. 32, no. 8, p. 1231-1240. DOI: 10.1109/4.604079

[15] VAISBAND, I., SAADAT, M., MURMANN, B. A closed-loop reconfigurable switched-capacitor DC-DC converter for sub-mW energy harvesting applications. IEEE Transactions on Circuits and Systems I: Regular Papers, 2015, vol. 62, no. 2, p. 385-394. DOI: 10.1109/TCSI.2014.2362971

[16] KIM, J., MOK, P. K. T., KIM, C. A $0.15 \mathrm{~V}$ input energy harvesting charge pump with dynamic body biasing and adaptive dead-time for efficiency improvement. IEEE Journal of Solid-State
Circuits, 2015, vol. 50, no. 2, p. 414-425. DOI: 10.1109/JSSC.2014.2375824

[17] PENG, H., TANG, N., YANG, Y., et al. CMOS startup charge pump with body bias and backward control for energy harvesting step-up converters. IEEE Transactions on Circuits and Systems I: Regular Papers, 2014, vol. 61, no. 6, p. 1618-1628. DOI: 10.1109/TCSI.2013.2290823

[18] DICKSON, J. F. On-chip high-voltage generation in MNOS integrated circuits using an improved voltage multiplier technique. IEEE Journal of Solid-State Circuits, 1976, vol. 11, no. 3, p. 374 to 378 . DOI: $10.1109 /$ JSSC.1976.1050739

[19] NINTANAVONGSA, P., MUNCUK, U., LEWIS, D. R., et al. Design optimization and implementation for RF energy harvesting circuit. IEEE Journal on Emerging and Selected Topics in Circuits and Systems, 2012, vol. 2, no. 1, p. 24-33. DOI: 10.1109/JETCAS.2012.2187106

[20] PAN, F., SAMADDAR, T. Charge Pump Circuit Design. McGraw Hill, 2006. (p. 95-96). ISBN: 007147045X, 978-0071470452

[21] TANZAWA, T. Design of DC-DC switched-capacitor voltage multiplier driven by DC energy transducer. In Proceedings of the 21st IEEE International Conference on Electronics, Circuits and Systems (ICECS). Marseille (France), 2014, p. 327-330. DOI: 10.1109/ICECS.2014.7049988

[22] HOQUE, M. R., AHMAD, T., MCNUTT, T. R., et al. A technique to increase the efficiency of high-voltage charge pumps. IEEE Transactions on Circuits and Systems II: Express Briefs, 2006, vol. 53, no. 5, p. 364-368. DOI: 10.1109/TCSII.2006.869922

\section{About the Author ...}

Davor VINKO was born in 1980 in Čakovec, Croatia. He received his M.Sc. and Ph.D. degree from J. J. Strossmayer University of Osijek, Faculty of Electrical Engineering, in 2005 and 2012, respectively. Since 2005 he has been affiliated with the Dept. of Communication, Faculty of Electrical Engineering, Computer Science and Information Technology, University of Osijek, where he reached a rank of assistant professor. His main research interests include analog circuit design, energy harvesting circuits and wirelessly powered devices.

\section{Appendix} values:

The crest factor $F$ is the ratio between peak and RMS

$$
F=\frac{\hat{U}}{U_{\mathrm{RMS}}}[-]
$$

With the defined duty cycle $D$ of the waveform (Fig. 2. and equation (7)), the crest factor $F$ becomes:

$$
F=\frac{\hat{U}}{D \cdot U_{\mathrm{RMS}}}[-]
$$

RMS value $U_{\text {RMS }}$ is defined as:

$$
U_{\mathrm{RMS}}=\sqrt{\frac{1}{T} \int_{0}^{T} u^{2}(t) \mathrm{d} t}[\mathrm{~V}],
$$


$U_{\mathrm{RMS}}=\sqrt{\frac{1}{\pi-2 \alpha} \int_{\alpha}^{\pi-\alpha}\left[U_{\mathrm{EH}} \sin (\theta)-U_{\mathrm{EH}} \sin (\alpha)\right]^{2} \mathrm{~d} \theta}[\mathrm{V}]$.

Integration is done for the time interval from $\alpha$ to $\pi-\alpha, \alpha$ corresponds to time interval $T_{\mathrm{OFF}}$ and is defined as:

$$
\begin{aligned}
& \alpha=\arcsin \left(\frac{U_{\text {th }}}{U_{\mathrm{EH}}}\right)\left[^{\circ}\right], \\
& U_{\mathrm{EH}} \sin (\alpha)=U_{\text {th }}[\mathrm{V}] .
\end{aligned}
$$

Considering (27), equation (25) changes to:

$$
U_{\mathrm{RMS}}=\sqrt{\frac{1}{\pi-2 \alpha} \int_{\alpha}^{\pi-\alpha}\left[U_{\mathrm{EH}} \sin (\theta)-U_{\mathrm{th}}\right]^{2} \mathrm{~d} \theta}[\mathrm{V}] .
$$

Solution for (28) is:

$$
U_{\mathrm{RMS}}=\sqrt{\frac{U_{\mathrm{EH}}^{2}}{2}+U_{\mathrm{th}}^{2}-\frac{3 U_{\mathrm{th}} U_{\mathrm{EH}}}{\pi-2 \alpha} \cos (\alpha)}[\mathrm{V}] .
$$

Using the following substitutions:

$$
\cos (\alpha)=\cos \left(\arcsin \left(\frac{U_{\text {th }}}{U_{\mathrm{EH}}}\right)\right)=\sqrt{1-\left(\frac{U_{\text {th }}}{U_{\mathrm{EH}}}\right)^{2}}
$$

the RMS value for section from $\alpha$ to $\pi-\alpha$ of a waveform given in Fig. 2b equals:

$$
U_{\mathrm{RMS}}=\sqrt{\frac{U_{\mathrm{EH}}^{2}}{2}+U_{\mathrm{th}}^{2}-\frac{3 U_{\mathrm{th}} U_{\mathrm{EH}} \sqrt{1-\left(\frac{U_{\mathrm{th}}}{U_{\mathrm{EH}}}\right)^{2}}}{\pi-2 \arcsin \left(\frac{U_{\mathrm{th}}}{U_{\mathrm{EH}}}\right)}}
$$

The same solution derives for time interval from $\pi+\alpha$ to $2 \pi-\alpha$. Then, the final expression for the crest factor $F$ of a waveform (Fig. 2b) is:

$$
F=\frac{U_{\mathrm{EH}}-U_{\mathrm{th}}}{D \cdot \sqrt{\frac{U_{\mathrm{EH}}^{2}}{2}+U_{\mathrm{th}}^{2}-\frac{3 U_{\mathrm{th}} U_{\mathrm{EH}} \sqrt{1-\left(\frac{U_{\mathrm{th}}}{U_{\mathrm{EH}}}\right)^{2}}}{\pi-2 \arcsin \left(\frac{U_{\mathrm{th}}}{U_{\mathrm{EH}}}\right)}}}[-] .
$$

Figure 6 compares the true crest factor $F$, (32), with the approximate crest factor, (8), for the waveform given by Fig. 2 b. For switch threshold voltage $U_{\text {th }}$ and energy harvester voltage $U_{\mathrm{EH}}$ ratio ranging from 0 to 0.95 , the error is below $4 \%$. In real life application, the $U_{\mathrm{th}} / U_{\mathrm{EH}}$ ratio would be below 0.5 , where the error of (8) is lower than $2 \%$. Therefore, the approximate crest factor given by (8) is sufficiently accurate.

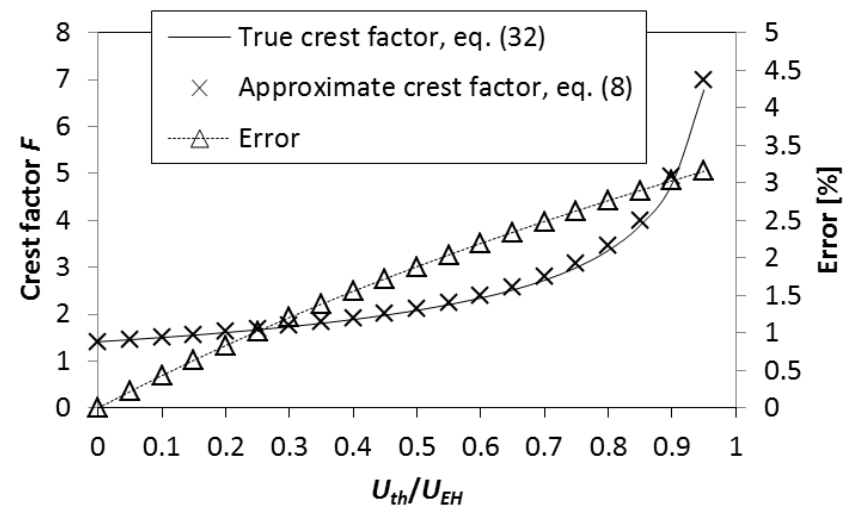

Fig. 6. Crest factor comparison. 